\title{
Correction to "Topological nonrealization results via the Goodwillie tower approach to iterated loopspace homology"
}

\author{
NiCHOLAS KUHN
}

\begin{abstract}
Manfred Stelzer has pointed out that part of Corollary 4.5 of [1] was not sufficiently proved, and, indeed, is likely incorrect as stated. This necessitates a little more argument to finish the proof of the main theorem of [1]. The statement of this theorem, and all the examples, remain unchanged.
\end{abstract}

55S10; 55S12, 55T20

In [1], the author showed that certain unstable modules over the mod 2 Steenrod algebra couldn't be realized as the reduced mod 2 cohomology of a space. The modules have the form $\Sigma^{n} M$, where $M$ is an unstable module of a special sort. The method of proof was to use a 2nd quadrant spectral sequence converging to $H^{*}\left(\Omega^{n} X ; \mathbb{Z} / 2\right)$ to show that, were a space $X$ to exist whose cohomology realized $\Sigma^{n} M, H^{*}\left(\Omega^{n} X ; \mathbb{Z} / 2\right)$ could not admit a cup product compatible with Steenrod operations.

The spectral sequence for $n>1$ is a newish one, arising from the Goodwillie tower of the functor $X \mapsto \Sigma^{\infty} \Omega^{n} X$, and Section 4 of [1] is devoted to collecting and proving some basic facts about this spectral sequence. I thank Manfred Stelzer for pointing out that part of Corollary 4.5 is likely over optimistic, and certainly was not sufficiently proved.

We assume notation as in [1].

In Corollary 4.5 , it was asserted that if $\tilde{H}^{*}(X ; \mathbb{Z} / 2) \simeq \Sigma^{n} M$ with $M$ unstable and also has no nontrivial cup products, then in the spectral sequence, one will have $E_{3}^{-1, *}=E_{2}^{-1, *}=E_{1}^{-1, *}$ and $E_{2}^{-2, *}=E_{1}^{-2, *}$. My mistake was in not adequately considering possible differentials on elements in $E_{1}^{-3, *}$ of the form $\sigma^{3} L_{n-1}(x \otimes y \otimes z)$. Under the hypotheses on the cup product, the $d_{1}$ differential on such terms will be 0 , by the same argument given explaining why $d_{1}$ is zero on terms of the form $L(x \otimes y)$ : by comparison to the classical Eilenberg-Moore spectral sequence. But there is no apparent reason why $d_{2}$ need also be zero on such terms. We can only conclude that $E_{2}^{-1, *}=E_{1}^{-1, *}$, and $E_{2}^{-2, *}=E_{1}^{-2, *}$.

Corollary 4.5 is used at one critical point in the proof of the main theorem given in Section 5. Lemma 5.3 asserts that a certain element in $E_{1}^{-1,2 d+2^{k+2}+1}$ is not a 
boundary. The argument given is that for dimension reasons, no $d_{r}$ for $r>2$ could have nonzero image in this bigrading. Implicit is that Corollary 4.5 takes care of $d_{1}$ and $d_{2}$. In light of the comments above, one needs a new argument for $d_{2}$.

It turns out that, except for one special case, a dimension argument still works: $E_{3}^{-3,2 d+2^{k+2}+2}$ contains no elements of the form $\sigma^{3} L_{n-1}(x \otimes y \otimes z)$. There are two extreme cases to consider: if $x, y$, and $z$ are all chosen from the top of $N_{0}$, and if $x$ and $y$ are chosen from the bottom of $N_{0}$ and $z$ is chosen from the bottom of $M_{1}$.

In the first case, $|x|=|y|=|z|=m+2^{k}$, and so $\sigma^{3} L_{n-1}(x \otimes y \otimes z)$ has bidegree $\left(-3,3 m+3 \cdot 2^{k}+2 n+1\right)$. In the second case, $|x|=|y|=d+2^{k}$ and $|z|=l+2^{k+1}$, and so $\sigma^{3} L_{n-1}(x \otimes y \otimes z)$ has bidegree $\left(-3,2 d+l+2^{k+2}+2 n+1\right)$.

We are assuming inequality (5-3), which says that $2^{k}>4 m-2 l+2 n-2$. One also has that $0 \leq l \leq d \leq m$ and $n \geq 1$. One can then check that, indeed,

$$
3 m+3 \cdot 2^{k}+2 n+1<2 d+2^{k+2}+2<2 d+l+2^{k+2}+2 n+1,
$$

unless we are in the special case $k=0, n=1, l=d=m=0$.

In this final special case, $n=1$, so we are trying to use the classical Eilenberg-Moore spectral sequence to show that, if $M$ is a $\mathbb{Z} / 2$ vector space concentrated in degree 0 , there cannot exist a space $X$ with $\widetilde{H}^{*}(X ; \mathbb{Z} / 2) \simeq \Sigma M \otimes \Phi(0,2)$, if all cup products are zero. Such a space will necessarily fit into a cofibration sequence of the form

$$
\bigvee S^{4} \rightarrow \bigvee \Sigma \mathbb{R} P^{2} \rightarrow X
$$

We leave it to the reader to check that, by appropriately including $S^{4}$ into the first wedge, and projecting out onto a $\Sigma \mathbb{R} P^{2}$ in the second wedge, one sees that $X$ will have a "subquotient" $Y$ with $\widetilde{H}^{*}(Y ; \mathbb{Z} / 2) \simeq \Sigma \Phi(0,2)$, and still with all cup products 0 .

Similar to, but simpler than, arguments in Section 6 of [1] (which dealt with $\Sigma^{2} \Phi(1,3)$ ), our arguments show that such a $Y$ can't exist. Repressing some suspensions from the notation, Figure 1 shows all of $E_{1}^{*, *}$ in total degree less than or equal to 4 , in the Eilenberg-Moore spectral sequence converging to $H^{*}(\Omega Y ; \mathbb{Z} / 2)$.

As cup products are assumed zero, $E_{2}^{*, *}=E_{1}^{*, *}$. Furthermore, $d_{2}(a \otimes a \otimes a)=0$ (and thus not $c$ ), because $a \otimes a \otimes a=(a \otimes a) * a$ and $d_{2}$ is a derivation with respect to the shuffle product $*$. Thus through degree $4, F^{-2} H^{*}(\Omega Y ; \mathbb{Z} / 2)$ would have a basis given by elements $1, \alpha, \beta, \delta, \epsilon_{1}, \epsilon_{2}, \gamma$, and $\omega$, in respective degrees $0,1,2,2$, $3,3,4$, and 4, and represented by $1, a, b, a \otimes a, a \otimes b, b \otimes a, c$, and $b \otimes b$. The structure of $\Phi(0,2)\left(\mathrm{Sq}^{1} a=b, \mathrm{Sq}^{2} b=c\right)$ shows that $\gamma=\beta^{2}=\alpha^{4}$. Furthermore, 


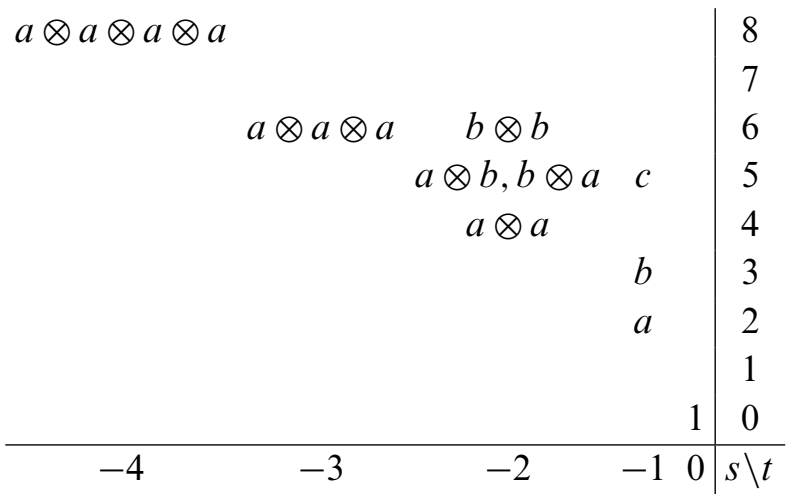

Figure 1: $E_{1}^{s, t}$ when $\widetilde{H}^{*}(Y ; \mathbb{Z} / 2) \simeq \Sigma \Phi(0,2)$

$\mathrm{Sq}^{1} \delta=\epsilon_{1}+\epsilon_{2}=\alpha \cup \beta$, as all three are represented by $a \otimes b+b \otimes a$. One then gets a contradiction, as

$$
0=\mathrm{Sq}^{1} \mathrm{Sq}^{1} \delta=\mathrm{Sq}^{1}(\alpha \cup \beta)=\beta^{2}=\gamma \neq 0 .
$$

We end by observing that $\widetilde{H}^{*}(\operatorname{SU}(3) / \operatorname{SO}(3) ; \mathbb{Z} / 2) \simeq \Sigma \Phi(0,2)$. Here, of course, cup products are not zero, due to Poincaré duality.

This research was partially supported by the National Science Foundation.

\section{References}

[1] N Kuhn, Topological nonrealization results via the Goodwillie tower approach to iterated loopspace homology, Algebr. Geom. Topol. 8 (2008) 2109-2129 MR2460881

Department of Mathematics, University of Virginia

Kerchof Hall, PO Box 400137, Charlottesville VA 22904-4137, USA

njk4x@virginia.edu

http://artsandsciences.virginia.edu/mathematics/people/faculty/ njk4x.html

Received: 5 November 2009 